\title{
Editorial: Annual Essay Competition and Workshop in India
}

\author{
Calvin Wai-Loon Ho ${ }^{1}$
}

Published online: 21 July 2018

(C) National University of Singapore and Springer Nature Singapore Pte Ltd. 2018

While there may not be a special theme to this instalment of the journal, I am pleased to present papers on two topics that have become mainstays: clinical ethics and research ethics. Regarding the former, our contributors have highlighted challenges including those that relate to alternative therapies, and the extent that the visiting policy in a healthcare facility's intensive care unit should be open. These are important topics in clinical ethics, and the discussions in these papers add to the rich dialogue that was presented in the previous thematic journal issue (volume 10; issue 1) on clinical ethics consultation. Research ethics is the other topic that the papers of this journal issue are concerned with. And more specifically, they attempt to address ethical and regulatory challenges that arise from rapid advancement in genetics and robotics, particularly those endowed with artificial intelligence. Importantly, this journal is keen to open up new dialogues on topics that have had a less conspicuous presence since the publication of our first volume in 2008. The book review presented in this journal issue is a harbinger of environment ethics; a topic that will underscore a prospective thematic journal issue currently scheduled for publication later in the year. It may perhaps be apparent that papers published in this journal are not drawn exclusively from contributors within Asia and the Pacific. To be sure, we continue to welcome the submission of manuscripts from all over the world, although themes discussed should be of interest to readers in these regions.

On a separate note, I am pleased to announce that our annual essay competition will be open on 1 August 2018. The essay may be centred on any theme within the field of bioethics pertinent to Asia and the Pacific, and must be in compliance with the following:

- The manuscript should be 6000 words or less in English, excluding essay title, cover page (free format) and references.

Calvin Wai-Loon Ho

medhwlc@nus.edu.sg

1 Centre for Biomedical Ethics, Yong Loo Lin School of Medicine, National University of

Singapore, 21 Lower Kent Ridge Rd, Singapore 119077, Singapore 
- References used should comprise no more than 100 citations.

- Authors must apply the journal's publication style (i.e. Chicago Manual of Style in the author-date system).

- The manuscript must be single-authored, original and not previously published in any medium.

In addition, an entrant must, as on 1 August 2018, be above 18 years of age and must fit any of the following profiles: (a) a registered student at a tertiary education institution or (b) an emerging scholar having been conferred a postgraduate academic degree (i.e. master's degree, $\mathrm{PhD}$ or equivalent degree) for not more than 4 years. All entries must be accompanied by a cover page to clearly indicate that the essay is to be entered into the competition, and submitted through Editorial Manager by 22 November 2018 (23:59 h GMT). Interested readers are welcome to visit the journal's website for more details about the competition.

Finally, I am pleased to inform readers that on 4 December 2018, the journal will be organising a case-writing workshop. This is to be conducted as a pre-congress event to the joint 14th World Congress of Bioethics of the International Association of Bioethics and 7th National Bioethics Conference of the Indian Journal of Medical Ethics. The conference is to be held from 5th to 7th December 2018, in Bengaluru, India. This workshop is intended to help emerging scholars develop skills in analysing cases in clinical ethics, research ethics, public health ethics, health policy and systems research, amongst other bioethics specialties, for publication as case commentaries in a bioethics journal. No registration fee is payable to participate in this workshop, but prior registration is required for both the workshop and the World Congress. For more information, please refer to webpage on pre-congress events at the congress website: http://ijme.in/nbc-20140321. While the workshop is directed at regional capacitybuilding for junior scholars, we also hope to mark the publication of the journal's 10 th volume through this and other events at the World Congress. If you are planning to be in Bengaluru, I very much look forward to meeting you. 